\title{
Prognostic Importance of Metastatic Site in Intermediate-risk Group Metastatic Renal Cell Cancer Treated with Tyrosine Kinase Inhibitors
}

\author{
Cengiz Karacin, Irem Bilgetekin, Fatma Bugdayci Basal and Omur Berna Oksuzoglu \\ Department of Medical Oncology, Dr Abdurrahman Yurtaslan Oncology Training and Research Hospital, Ankara, Turkey
}

\begin{abstract}
Objective: To determine the prognostic importance of the metastatic site in metastatic renal cell cancer (mRCC) patients in the International Metastatic Renal Cell Carcinoma Database Consortium (IMDC) intermediate-risk.

Study design: Observational study.

Place and Duration of Study: Department of Medical Oncology, Dr Abdurrahman Yurtaslan Oncology Training and Research Hospital, Ankara, Turkey, from January 2010 to November 2018.

Methodology: Records of 113 mRCC patients, determined to be in the intermediate-risk group according to IMDC criteria, were reviewed retrospectively. All patients used a tyrosine kinase inhibitor - sunitinib or pazopanib - for metastatic disease. Patients' records included age, gender, metastatic site, number of metastases and treatment regimen. The Kaplan-Meier method was used for survival analysis, and a Cox regression model was formed.

Results: The median age of the patients was 58 years (Q1 - Q3: 44 - 66 years) and $87.6 \%$ of the patients had $\geq 2$ metastatic sites. The most common metastatic sites were the lung $(51.3 \%)$, lymph nodes $(26.5 \%)$, bone $(26.5 \%)$ and brain $(17.7 \%)$. Median overall survival (OS) was shorter in the patients with bone and brain metastasis than in those without ( 15.0 months vs. 21.0 months, $p=$ .026 and 14.0 months vs. 21.0 months, $p=.009$, respectively). Multivariate analysis showed that brain and bone metastasis were independent prognostic risk factors (HR: 2.43, $p=.017$ and HR: 2.10, $p=.042$, respectively).

Conclusion: Bone and brain metastasis had a negative effect on OS in IMDC intermediate-risk group mRCC patients.

Key Words: Metastatic site, Brain metastasis, Bone metastasis, Renal cell carcinoma, Prognosis.

How to cite this article: Karacin C, Bilgetekin I, Basal FB, Oksuzoglu OB. Prognostic Importance of Metastatic Site in Intermediate-risk Group Metastatic Renal Cell Cancer Treated with Tyrosine Kinase Inhibitors. J Coll Physicians Surg Pak 2020; 30(06):590-594 https://doi.org/10.29271/jcpsp.2020.06.590.
\end{abstract}

\section{INTRODUCTION}

Renal cell cancer (RCC) constitutes approximately $3 \%$ of all cancers in adults. ${ }^{1}$ Among RCC patients, $15 \%-20 \%$ present with metastatic disease (metastatic RCC [mRCC]) and the 5-year survival rate does not exceed $12 \% .{ }^{2}$ In approximately $90 \%$ of cases, VHL tumour suppressor gene mutation and elevated expression of vascular endothelial growth factor (VEGF) play a role in the pathogenesis. ${ }^{3}$

There are several prognostic risk scoring systems for $\mathrm{mRCC}^{4}{ }^{4}$ and the International Metastatic Renal Cell Carcinoma Database Consortium (IMDC) system is among the most commonly used. ${ }^{4}$ IMDC scoring classifies patients into three risk groups: favourable risk, intermediate-risk, and poor risk. ${ }^{4}$

Correspondence to: Cengiz Karacin, Deptartment of Medical Oncology, Dr Abdurrahman Yurtaslan Oncology

Training and Research Hospital, Ankara, Turkey

E-mail: cengizkaracin@yahoo.com

Received: May 06, 2020; Revised: June 20, 2020;

Accepted: July 02, 2020

DOI: https://doi.org/10.29271/jcpsp.2020.06.590
According to the IMDC scoring system, approximately $50 \%$ of $\mathrm{mRCC}$ patients are in the intermediate-risk group. ${ }^{4}$ Patients in this group are heterogeneous in terms of overall survival (OS). ${ }^{5}$ However, to date, no marker has been found that predicts OS in this patient group.

Studies show that the metastatic site in mRCC patients could be prognostic. ${ }^{6-8}$ Just as in the case of other solid organ tumours, OS is significantly shorter in $\mathrm{MRCC}$ patients with brain metastasis. ${ }^{9}$ Other studies report that bone metastasis is a marker of poor prognosis in mRCC patients. ${ }^{6,7}$ There are insufficient data in the literature on the prognostic effect of metastatic sites in $\mathrm{MRCC}$ patients in the IMDC intermediate-risk group. In addition, according to IMDC, the intermediate-risk group is also the most heterogeneous in terms of survival. Therefore, the present study aimed to determine the prognostic importance of the metastatic site in $\mathrm{MRCC}$ patients in the IMDC intermediate-risk group.

\section{METHODOLOGY}

This observational study retrospectively reviewed the records of 113 mRCC patients classified as belonging to the IMDC intermediate-risk group who were admitted to the medical oncology clinic 
from January 2010 to November 2018. The study was approved by the Institutional Ethical Review Board. Inclusion criteria were age $\geq 18$ years, a histologically confirmed diagnosis of clear cell RCC, metastatic disease according to response evaluation criteria in solid tumours v. 1.1 (RECIST v 1.1), and use of a tyrosine kinase inhibitor (TKI) - sunitinib or pazopanib. Patients were excluded from the study if they had histopathologies other than clear cell carcinoma or if their data were incomplete.

Data were retrieved from the hospital patient records and information system. They included age, gender, metastatic site, number of metastases, treatment regimen, surgical details, IMDC risk score parameters, the date of the last follow-up and the date of death. OS was defined as the time from initiation of treatment to death due to any cause.

The IMDC risk scoring system includes two clinical and four laboratory criteria namely the time from diagnosis to systemic treatment <1 year; Karnofsky performance status $<80 \%$; haemoglobin value below normal; calcium level above the upper limit of normal; neutrophilia; and thrombocytosis. Patients are classified in the favourable-risk group if none of these criteria is met, in the intermediate-risk group if 1 or 2 are met, and the poor-risk group if $\geq 3$ are met. ${ }^{4}$

Data were analysed using IBM SSPS Statistics for Windows version 20.0 software (IBM Corp., Armonk, NY). Qualitative variables were expressed as frequencies and percentages; quantitative variables as median (IQR or interquartile range). The conformity of numerical data to a normal distribution was assessed using the Kolmogorov-Smirnov test. OS was determined using the Kaplan-Meier method, and the log-rank test was used for univariate comparison. A multivariate Cox regression model was used to identify independent risk factors. All statistical analyses were two-way and the level of statistical significance wasset at $p<.05$.

\section{RESULTS}

The study included 113 IMDC intermediate-risk group mRCC patients, $73.5 \%$ males and $26.5 \%$ females with a median age of 58 years (Q1-Q3: 44-66 years). The Karnofsky performance score was $\geq 80$ in $77.9 \%$ of the patients. Histologically, the sarcomatoid differentiation rate was $8.8 \%$. Primary nephrectomy was observed in $82.3 \%$ of the patients and $\geq 2$ metastatic sites were noted in 99 (87.6\%) of the patients. Interferon- $\alpha$ (IFN- $\alpha$ ) had been used by $73 \%$ of the patients, with a median duration of use of 2 months (Q1-Q3: 1-2 months). The most common metastatic site was the lungs (51.3\%), followed by the lymph nodes $(26.5 \%)$, bones $(26.5 \%)$ and brain $(17.7 \%)$. The demographic and clinicopathological characteristics of the patients are shown in Tablel.

Median follow-up was 19.0 months (IQR: 3.0 - 51.0 months). Median OS for all patients was 19 months (95\% Cl: 15.2-22.8 months). Median OS was shorter in the patients with bone metastasis than in those without (15.0 months vs. 21.0 months, $p=.026$, Figure 1 ). Median OS was shorter in the patients with brain metastasis than in those without (14.0 months vs. 21.0 months, $p=.009$, Figure 2). OS was longer in the patients with lung metastasis than in those without (22.0 months vs. 17.0 months, $p=.023$ ). Except for bone, brain, and lung metastasis, there were not any other factors that affected OS, based on univariate analysis. The results of univariate analysis of the factors affecting OS are shown in Tablell.

The factors associated with OS, based on univariate analysis, were included in a multivariate Cox regression model. The multivariate analysis showed that brain and bone metastasis were independent prognostic factors (HR: 2.43, 95\% Cl: 1.17-5.02, $p$ $=.017$, and HR: $2.10,95 \% \mathrm{Cl}: 1.03-4.30, p=.042$, respectively, Table III).

Table I: Demographic and clinicopathological characteristics of the patients $(n=113)$.

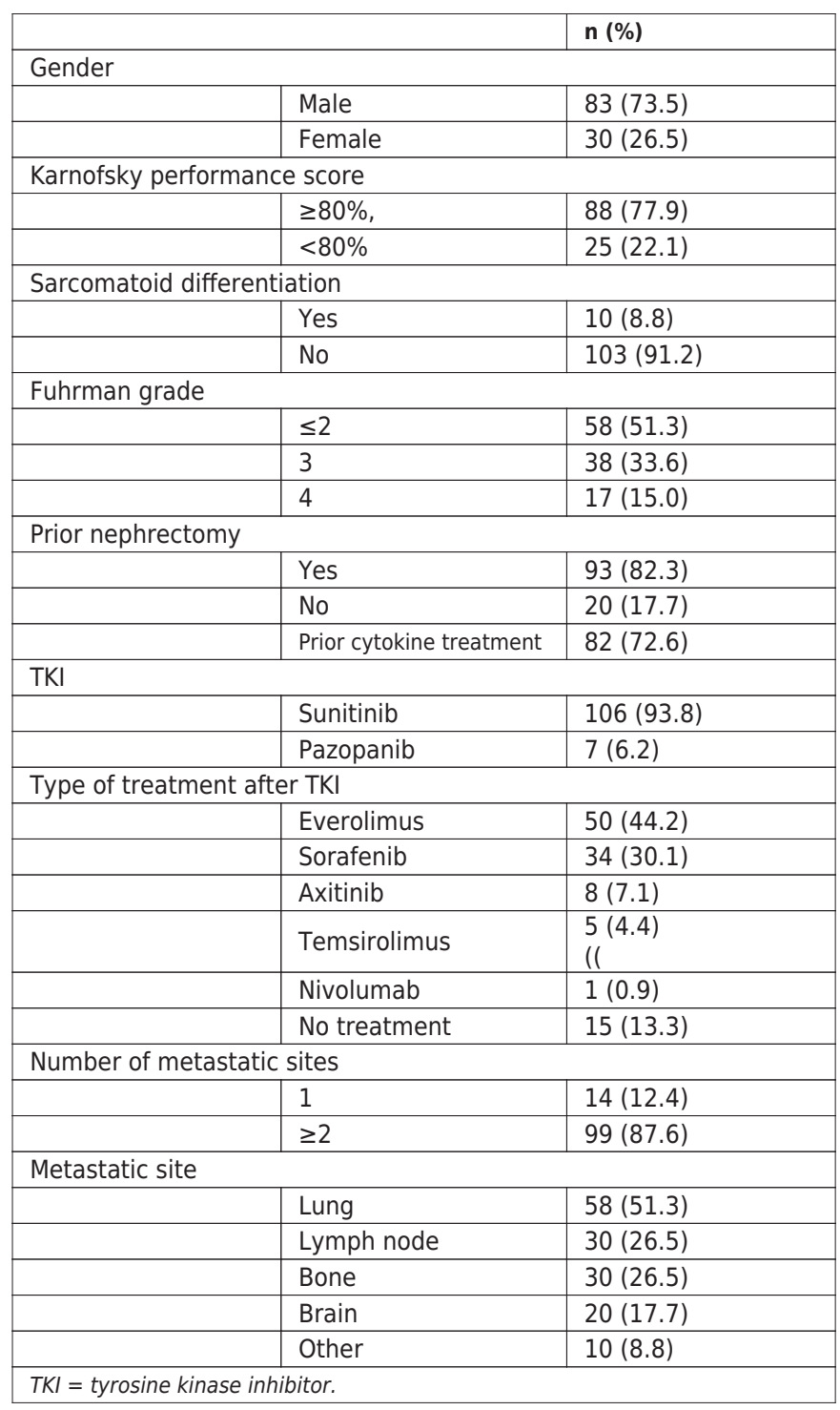

Table II: Univariate analysis of factors affecting overall survival.

\begin{tabular}{|l|c|c|}
\hline & $\begin{array}{c}\text { Median OS, months } \\
(\mathbf{9 5 \%} \mathbf{C l})\end{array}$ & p \\
\hline Karnofsky performance score & \multicolumn{2}{|l}{} \\
\hline
\end{tabular}




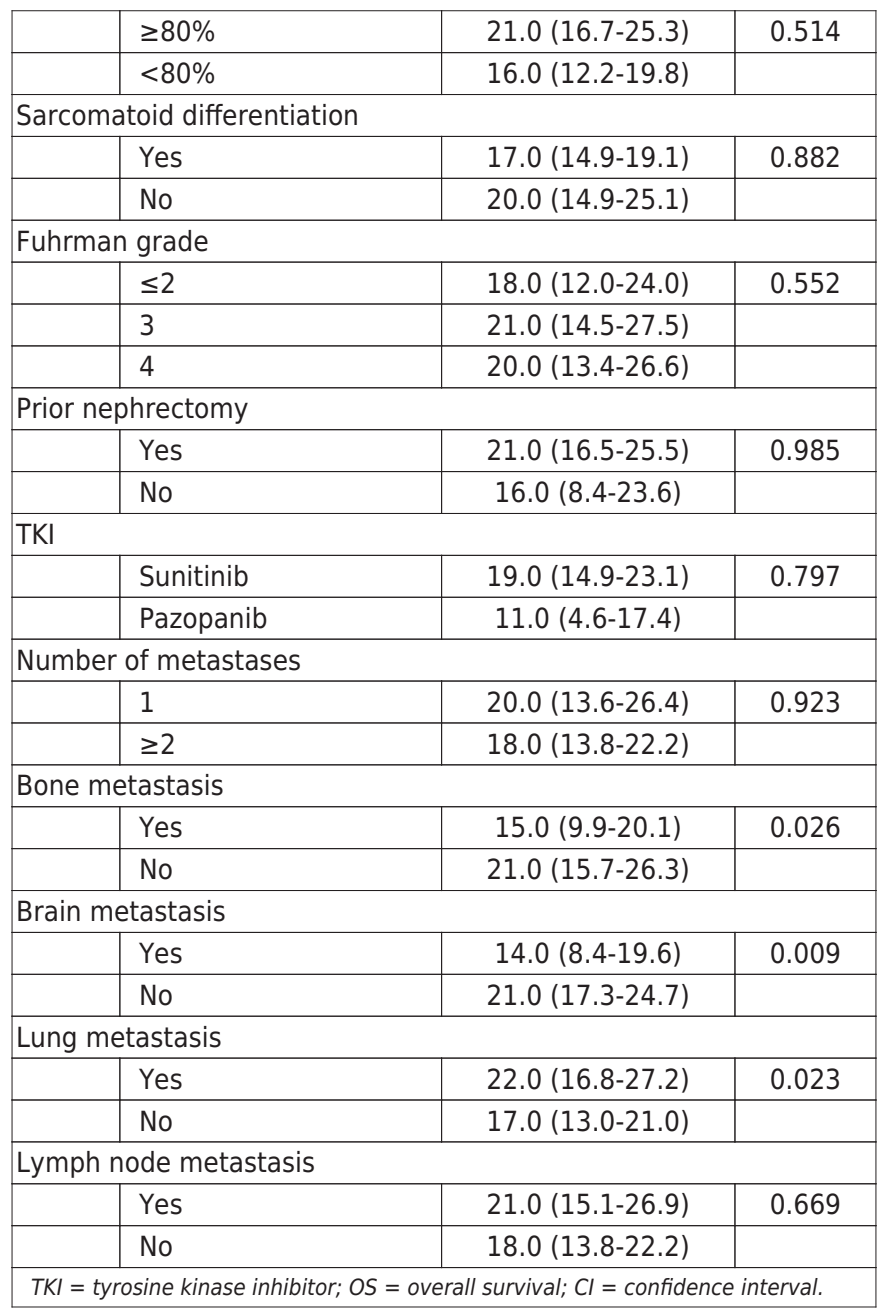

Table III: Multivariate analysis of the effect of bone, brain, and lung metastasis on overall survival.

\begin{tabular}{|c|c|c|}
\hline & HR $(95 \% \mathrm{Cl})$ & $\mathbf{p}$ \\
\hline \multicolumn{3}{|l|}{ Bone metastasis } \\
\hline No & Reference & 0.042 \\
\hline Yes & $2.10(1.03-4.30)$ & \\
\hline \multicolumn{3}{|l|}{ Brain metastasis } \\
\hline No & Reference & 0.017 \\
\hline Yes & $\begin{array}{l}2.43 \\
(1.17-5.02)\end{array}$ & \\
\hline \multicolumn{3}{|l|}{ Lung metastasis } \\
\hline No & Reference & 0.582 \\
\hline Yes & $\begin{array}{l}1.23 \\
(0.59-2.54)\end{array}$ & \\
\hline
\end{tabular}

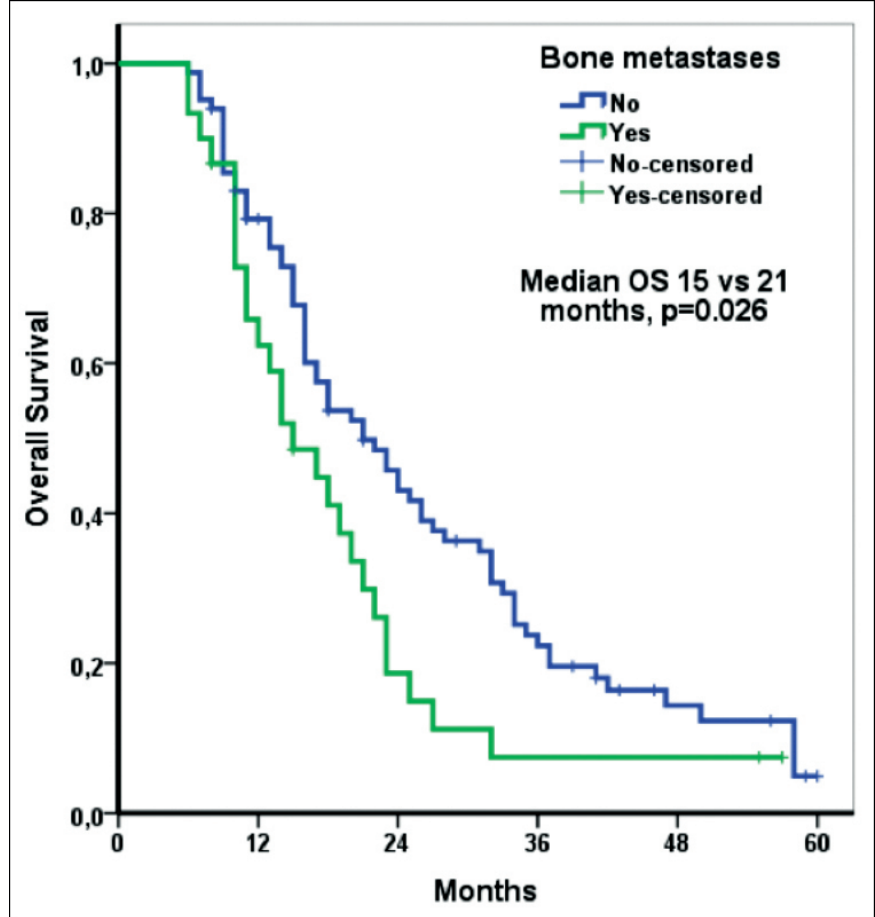

Figure 1: Overall survival according to bone metastasis.

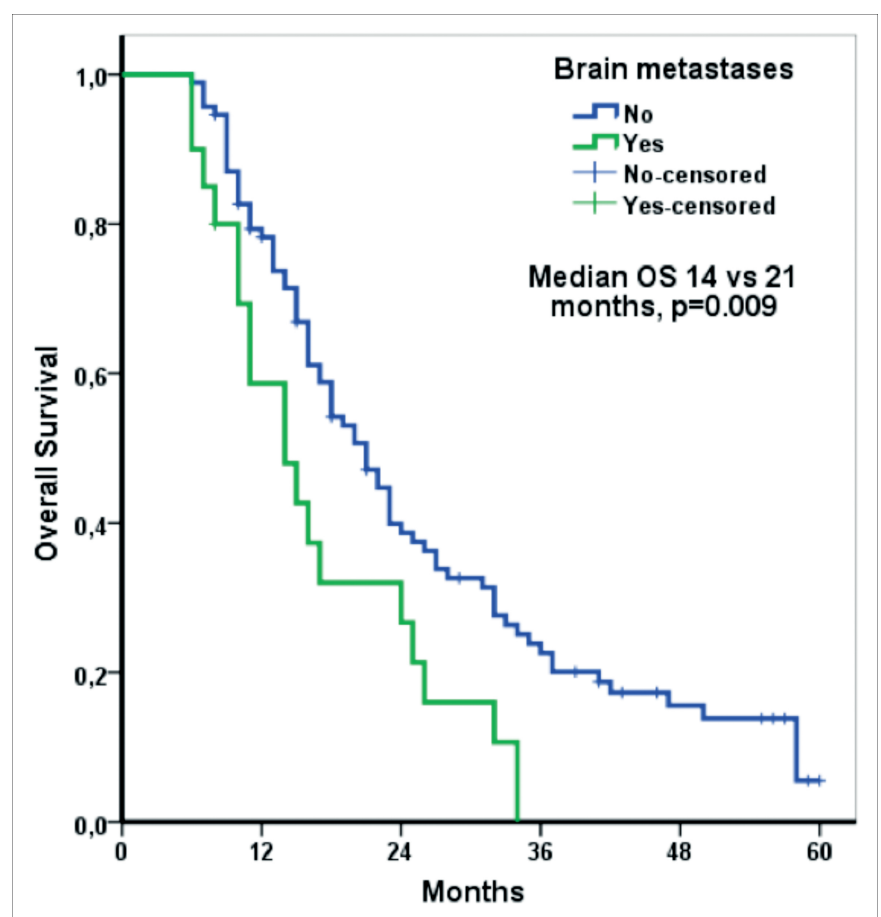

Figure 2: Overall survival according to brain metastasis.

\section{DISCUSSION}

The findings of this study show that brain and bone metastasis are independent prognostic factors in $\mathrm{MRCC}$ patients in the IMDC intermediate-risk group and that the presence of either of these two metastatic sites has a negative effecton OS.

Numerous studies have evaluated the prognostic importance of the metastatic site in mRCC patients; and bone metastasis, in particular, was shown to be a factor associated with poor prog- 
nosis. ${ }^{6-8,10,11}$ Negrier et al. studied $782 \mathrm{mRCC}$ patients that received cytokine-based treatment and reported that bone metastasis was an independent prognostic factor. ${ }^{12}$ Median OS in their patients without bone metastasis was longer than in those with bone metastasis (15.7 vs. 8.8 months). ${ }^{12}$ Similar results have been noted in $\mathrm{mRCC}$ patients treated with TKIs. Patil et al. ${ }^{7}$ compared sunitinib and IFN-a as first-line treatments for $\mathrm{mRCC}$. They reported that bone metastasis was a prognostic factor for OS in both the sunitinib and IFN-a arms. McKay et al. ${ }^{6}$ evaluated the prognostic importance of the metastatic site in mRCC patients grouped according to IMDC risk classification. They observed that bone metastasis was a factor associated with poor prognosis in all three groups. Median OS in their IMDC intermediate-risk group $\mathrm{mRCC}$ patients with bone metastasis was 17.0 months, compared to 23.6 months in those without bone metastasis $(p<.001) .{ }^{6}$ Similarly in the present study, the median OS in IMDC intermediate-risk group $\mathrm{MRCC}$ patients with bone metastasis was shorter than in those without bone metastasis (15.0 months vs. 21.0 months, $p=.026$ ). Moreover, multivariate analysis showed that bone metastasis was an independent prognostic factor.

The mechanism by which bone metastasis negatively affects OS remains to be fully understood. Preclinical studies have shown that changes in the bone micro-environment might be a cause of both primary tumour growth and formation of metastases beyond bone. ${ }^{13}$ Another preclinical study reported that there might be a relationship between osteoblasts from bone cells and distant metastasis beyond bone and that tumour volume decreased as the number of osteoblasts decreased. ${ }^{14}$ Although a phase II study showed that treatment of bone metastasis with zoledronic acid together with everolimus contributed to extending OS in mRCC patients, there are only a few retrospective in vivostudies that report a similar finding. ${ }^{15-17}$

The majority of relevant clinical trials have not included patients with brain metastasis, and they have not yielded sufficient data related to brain metastasis. ${ }^{18}$ Therefore, in vivo studies that generate data related to brain metastasis are of great importance. There are very few studies on the prognostic importance of brain metastasis in mRCC patients. ${ }^{8,10,19}$ Atzpodien etal. ${ }^{8}$ evaluated mRCC patients given cytokine-based treatment, and they observed that there was not a relationship between brain metastasis and OS. ${ }^{8}$ However, the number of patients with brain metastasis in that study was not sufficient for evaluating the prognostic importance of brain metastasis.. Mekhail et al. studied 353 mRCC patients and reported that median OS was 8.4 months in patients with brain metastasis, versus 14.8 months in those without brain metastasis. ${ }^{10}$ However, the difference was not significant - probably because of the low rate $(4 \%)$ of brain metastasis. A study performed with data from the surveillance, epidemiology, and end results (SEER) database reported that brain metastasis at the time of the diagnosis of RCC was an independent prognostic factor. ${ }^{19}$ The percentage of $\mathrm{mRCC}$ patients with brain metastasis in the present study was higher (17.7\%) than in other studies. ${ }^{8,10}$ In this study, median OS in the mRCC patients with brain metastasis was 14.0 months, versus 21.0 months in those without brain metastasis ( $p=$ .009). Moreover, multivariate analysis showed that brain metastasis was an independent prognostic factor in $\mathrm{MRCC}$ patients in the IMDC intermediate-risk group.

Most RCC clinical trials have not included patients with brain metastasis, which occurs in $4 \%-17 \%$ of advanced-stage RCC patients and negatively affects OS. ${ }^{20}$ Although the pathophysiology of the negative effect of brain metastasis on OS is not fully known, intracranial haemorrhaging can occur because of the vascular structure of tumours. ${ }^{21}$ The relationship between the brain micro-environment and metastatic tumour cells has not been fully elucidated because of the difficulty of in vitro modelling. ${ }^{9}$ Research has shown that in mRCC patients, brain metastasis, especially if there are $\geq 2$ metastatic lesions in the brain, is associated with poor prognosis. ${ }^{22}$

There are studies on the importance of bone metastases in mRCC patients treated with TKI, but in this research is the first that evaluated both brain and bone metastases and demonstrated prognostic significance. Beuselinck et al. and Patil et al. did not evaluate the metastatic sites according to IMDC risk groups in their studies. McKay et al. evaluated bone metastasis according to IMDC, but they did not provide any information about brain metastasis.

The present study has some limitations. As data related to bisphosphonate or local treatment of bone metastasis were not obtained, the effects of these treatments on prognosis could not be evaluated. In addition, data were not obtained about the number of metastases in the brain, whether brain metastasis was symptomatic, and local treatments of brain metastasis, all of which can affect prognosis. Last, due to the small number of mRCC patients with liver metastasis, the prognostic importance of liver metastasis could not be evaluated.

\section{CONCLUSION}

The results of this study suggest that bone and brain metastasis may negatively affect OS in MRCC patients in the IMDC intermediate-risk group. Based on these findings, risk stratification, according to the metastatic site, can be used in $\mathrm{MRCC}$ patients in the IMDC intermediate-risk group. Larger-scale comparative studies that include mRCC patients in the IMDC poor-risk and favourable-risk groups might lead to the integration of the metastatic site into the IMDC RCC risk scoring system. When planning clinical trials, the metastatic site should be included in patient stratification, in addition to the classic IMDC risk groups.

\section{ETHICALAPPROVAL:}

Ethics Committee approval was received from the Ethics Committee of University of Health Sciences, Dr Abdurrahman Yurtaslan Oncology Training and Research Hospital (Number:E-91, April 4, 2020).

\section{PATIENTS' CONSENT:}

Informed consents were obtained from all participants or their family. 


\section{CONFLICT OF INTEREST:}

Authors declared no conflict of interest.

\section{AUTHORS' CONTRIBUTION:}

CK: Conception and design, analysis and interpretation of data, drafting of manuscript.

IB: Interpretation of data.

FBB: Acquisition of data.

OBO: Conception and design, reviewed the paper, advised and final approval.

\section{REFERENCES}

1. Bray F, Ferlay J, Soerjomataram I, Siegel RL, Torre LA, Jemal A. Global cancer statistics 2018: GLOBOCAN estimates of incidence and mortality worldwide for 36 cancers in 185 countries. CA Cancer J Clin 2018; 68(6):394-424.

2. Howlader NA, Krapcho M, Miller D, Brest A, Yu M, Ruhl J, et al. SEER cancer statistics review, 1975-2016. National Cancer Institute 2016.

3. Rathmell WK, Chen S. VHL inactivation in renal cell carcinoma: Implications for diagnosis, prognosis and treatment. Expert Rev Anticancer Ther 2008; 8(1):63-73.

4. Heng DY, Xie W, Regan MM, Warren MA, Golshayan AR, Sahi C, et al. Prognostic factors for overall survival in patients with metastatic renal cell carcinoma treated with vascular endothelial growth factor-targeted agents: Results from a large, multicenter study. J Clin Oncol 2009; 27(34): 5794-9.

5. Procopio G, Bamias A, Schmidinger M, Hawkins R, Sánchez AR, Estevez SV, et al. Real-world effectiveness and safety of pazopanib in patients with intermediate prognostic risk advanced renal cell carcinoma. Clin Enitourinary Cancer 2019; 17(3):e526-e33.

6. McKay RR, Lin X, Perkins JJ, Heng DY, Simantov R, Choueiri TK. Prognostic significance of bone metastases and bispho-sphonate therapy in patients with renal cell carcinoma. Eur Urolo 2014; 66(3):502-9.

7. Patil S, Figlin R, Hutson T, Michaelson M, Négrier S, Kim S, et al. Prognostic factors for progression-free and overall survival with sunitinib targeted therapy and with cytokine as first-line therapy in patients with metastatic renal cell carcinoma. Ann Oncol 2011; 22(2): 295-300.

8. Atzpodien J, Royston P, Wandert T, Reitz M. Metastatic renal carcinoma comprehensive prognostic system. $\mathrm{Br}$ J Cancer 2003; 88(3):348-53.

9. Lowery FJ, Yu D. Brain metastasis: Unique challenges and open opportunities. Biochim Biophys Acta Rev Cancer 2017; 1867(1):49-57.

10. Mekhail TM, Abou-Jawde RM, Boumerhi G, Malhi S, Wood $L$, Elson $P$, et al. Validation and extension of the memorial sloan-kettering prognostic factors model for survival in patients with previously untreated metastatic renal cell carcinoma. J Clin Oncol 2005; 23(4):832-41.

11. Motzer RJ, Escudier B, Oudard S, Hutson TE, Porta C, Bracarda $S$, et al. Phase 3 trial of everolimus for metastatic renal cell carcinoma: Final results and analysis of prognostic factors. Cancer 2010; 116(18):4256-65.

12. Négrier S, Escudier B, Gomez F, Douillard J, Ravaud A, Chevreau $C$, et al. Prognostic factors of survival and rapid progression in 782 patients with metastatic renal carcinomas treated by cytokines: A report from the groupe français d'immunothérapie. Ann Oncolo 2002; 13(9): 1460-8.

13. Fornetti J, Welm AL, Stewart SA. Understanding the bone in cancer metastasis. J Bone Miner Res 2018; 33(12): 2099-113.

14. Engblom C, Pfirschke C, Zilionis R, Martins JDS, Bos SA, Courties $\mathrm{G}$, et al. Osteoblasts remotely supply lung tumors with cancer-promoting SiglecFhigh neutrophils. Science 2017; 358 (6367): eaal5081.

15. Broom RJ, Hinder V, Sharples K, Proctor J, Duffey S, Pollard $\mathrm{S}$, et al. Everolimus and zoledronic acid in patients with renal cell carcinoma with bone metastases: A randomised first-line phase II trial. Clin Genitourin Cancer 2015; 13(1): 50-8.

16. Beuselinck B, Wolter P, Karadimou A, Elaidi R, Dumez H, Rogiers $A$, et al. Concomitant oral tyrosine kinase inhibitors and bisphosphonates in advanced renal cell carcinoma with bone metastases. BrJ Cancer 2012; 107(10):1665-71.

17. Keizman D, Ish-Shalom M, Pili R, Hammers $H$, Eisenberger MA, Sinibaldi V, et al. Bisphosphonates combined with sunitinib may improve the response rate, progression free survival and overall survival of patients with bone metastases from renal cell carcinoma. Eur J Cancer 2012; 48(7):1031-7.

18. Motzer RJ, Hutson TE, Cella D, Reeves J, Hawkins R, Guo J, et al. Pazopanib versus sunitinib in metastatic renal-cell carcinoma. N Engl J Med 2013; 369:722-31.

19. Chandrasekar T, Klaassen Z, Goldberg H, Kulkarni GS, Hamilton RJ, Fleshner NE. Metastatic renal cell carcinoma: Patterns and predictors of metastases-a contemporary population-based series. Urolo Oncol 2017; 35(11): 661.e7-661.e14.

20. Sheehan JP, Sun MH, Kondziolka D, Flickinger J, Lunsford LD. Radiosurgery in patients with renal cell carcinoma metastasis to the brain: Long-term outcomes and prognostic factors influencing survival and local tumor control. J Neurosurg 2003; 98(2):342-9.

21. Bitoh S, Hasegawa H, Ohtsuki H, Obashi J, Fujiwara M, Sakurai M. Cerebral neoplasms initially presenting with massive intracerebral hemorrhage. Surg Neurol 1984; 22(1):57-62.

22. Suarez-Sarmiento Jr A, Nguyen KA, Syed JS, Nolte A, Ghabili $\mathrm{K}$, Cheng $\mathrm{M}$, et al. Brain metastasis from renal-cell carcinoma: An institutional study. Clin Gen Cancer 2019; 17: el163-e70. 